\title{
Lumboperitoneal Shunt - A Simple Answer to Idiopathic Intracranial Hypertension
}

KHANDKAR ALI KAWSAR ${ }^{1}$

\begin{abstract}
:
Idiopathic Intracranial Hypertension commonly can present in young women. It is a relatively common disorder but unfortunately under diagnosed. Surgery can be an option of treatment for these patients. Here we report a can not a young girl presenting with IIH, successfully treated by a shunt surgery.
\end{abstract}

Keyword: Idiopathic intracranial hypertension, shunt, Bangladesh.

\section{Introduction:}

The syndrome of increased intracranial pressure (ICP) without ventriculomegaly or mass lesion, and with normal cerebrospinal fluid (CSF) composition, was first described more than a century ago. ${ }^{1}$ Often referred to as "pseudotumor cerebri" but more appropriately called "idiopathic intracranial hypertension" (IIH), it is a surprisingly common disorder. In young overweight women, the annual incidence is as high as 20 per 100,000 persons. ${ }^{2}$ It is often difficult to manage. Here we present a case of 22-year female, who presented with IIH and successfully treated with lumboperitoneal shunt. To our knowledge, this is the first reported case of IIH to be treated with lumbo-peritoneal shunt in Bangladesh.

\section{Case report:}

A 22-year old female presented to us with the complaint of headache, which was mild for 2 months but for last 7 days, it became severe. It involved all of her head and aggravated on straining, coughing and bending forward. It relieved at first by taking analgesics. It aggravated in the morning but
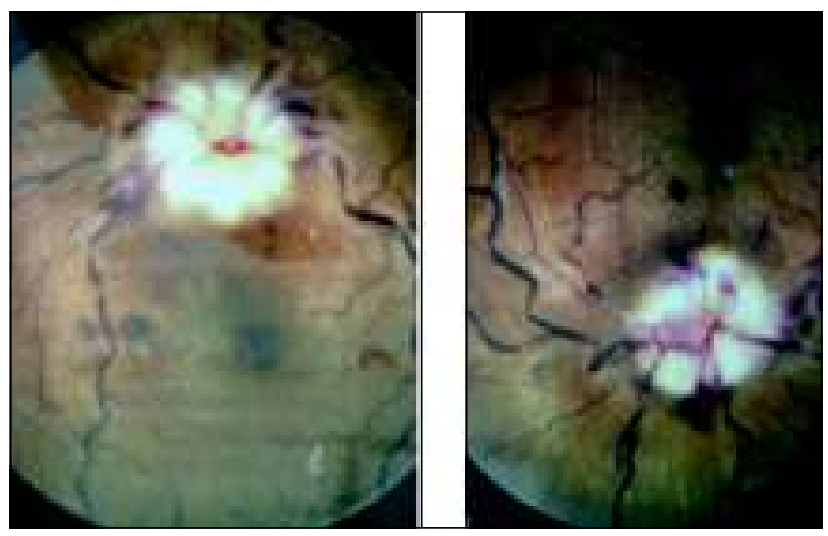

Fig.-1: Fundoscopic picture of the patient revealing bilateral papilloedema.

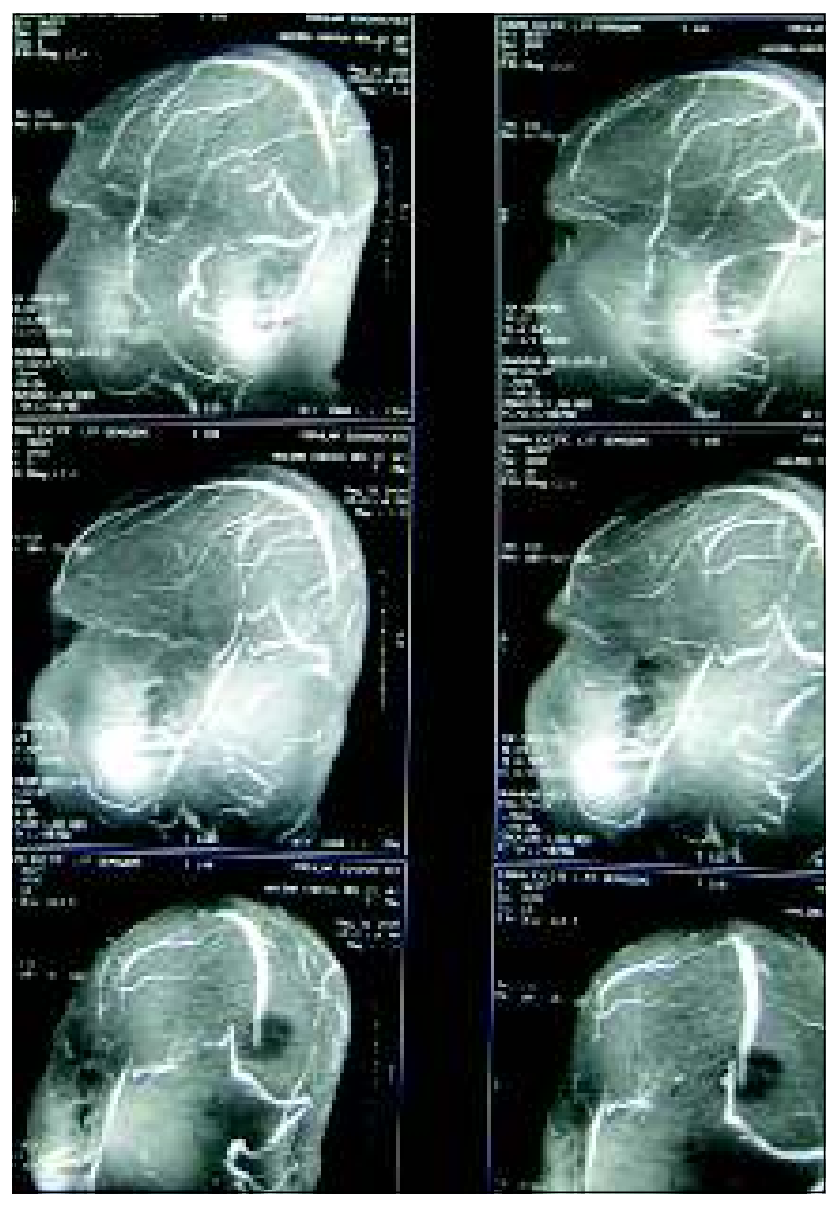

Fig.-2: Magnetic Resonance Venogram (MRV) scan showing no abnormality in venous sinuses.

later on it became severe throughout the day. The headache was associated with vomiting, which was spontaneous and projectile in nature. She also complained of blurring of vision for last 7 days. There was no history of fever, seizures, loss of consciousness.

1. Junior Consultant (Current Charge), Akhaura, Brahmanbaria

Correspondence : Dr. Khandkar Ali Kawsar, Junior Consultant (Current Charge), Akhaura, Brahmanbaria 
On examination, she was mildly obese. She was conscious and well oriented to time, place and person. Her vitals along with higher mental functions were normal and cranial nerves intact. Her systemic examinations were normal and no evidence of focal neurological deficit except bilateral papilloedema on fundoscopy. Haematology and biochemistry were within normal limits. Magnetic Resonance Imaging (MRI) and Magnetic Resonance Venogram (MRV) of brain revealed no abnormality. Then the diagnosis of idiopathic intracranial hypertension (IIH) was reached.

Lumbar puncture was done, in which, high CSF pressure was noted. Patient got relieved from her headache. But it reappeared soon. Then we put a lumbar drain for 5 days. But, when it was blocked, the headache reappeared. So, we decided to treat her with lumboperitoneal shunt. The shunt was inserted at the level of $4^{\text {th }}$ lumbar vertebra and secured with the lamina of that vertebra. The abdominal end was tunneled through the subcutaneous tract into the anterior abdominal wall. The peritoneal cavity was opened and the abdominal end was inserted. The wound was closed in layers. The patient, from the first postoperative day, was free from headache. She gradually regained her vision also. One-year follow-up revealed complete recovery from her IIH.

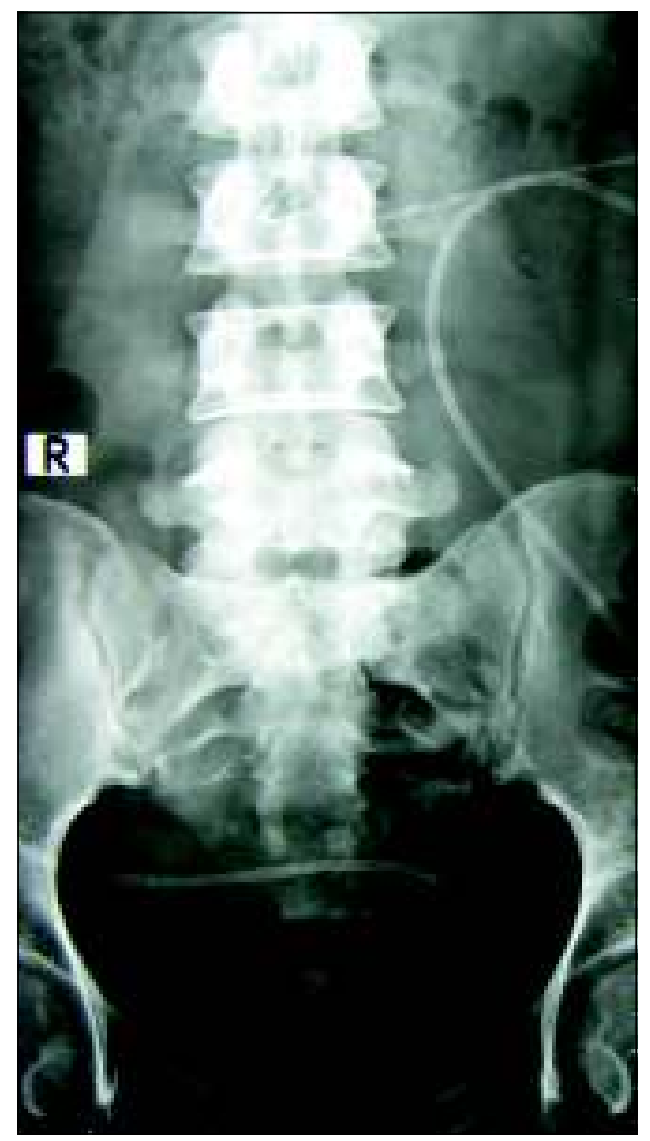

Fig.-3: Plain X-ray of lumbosacral region showing the lumboperitoneal shunt.

\section{Discussion:}

Benign intracranial hypertension was first described by Quincke in 1896, but the cause of this disorder continues to be a source of speculation. The prevalence of benign intracranial hypertension has been estimated to range from 1 to 19 cases per 100,000 population. ${ }^{2}$ The pathophysiologic basis of benign intracranial hypertension remains unclear but is associated with the conditions such as aberrations in intracranial volume regulation, metabolic diseases, medication-associated abnormalities and miscellaneous disorders. $^{3}$

The definition of IIH has evolved with clinical experience and advances in imaging technology. Currently, IIH can be diagnosed only if the following criteria are met : 1) symptoms and signs attributable to increased ICP or papilledema; 2) elevated ICP recorded during lumbar puncture in the lateral decubitus position; 3) normal CSF composition; 4) no imaging evidence of ventriculomegaly or a structural cause for increased ICP, such as a brain parenchymal, ventricular, meningeal, or venous sinus abnormality; and 5) no other cause of intracranial hypertension identified, such as use of certain medications. ${ }^{4}$

Diagnosis is primarily clinical and requires radiographic exclusion of an intracranial mass and measurement of cerebrospinal fluid pressure. In our case, we did MRI, MRV and then came to this diagnosis.

Treatment is directed at reducing intracranial pressure in idiopathic cases or correcting associated conditions. Not all patients with IIH require treatment. After establishing the diagnosis, asymptomatic individuals with normal vision and minimal papilloedema can be monitored frequently for the development of symptoms or visual decline. A small percentage of patients improve after their diagnostic lumbar puncture (LP). Therapy is initiated in the presence of visual acuity or visual field loss (apart from mild enlargement of the blind spot), moderate to severe (Frisén grade 3-5) papilledema or persistent headaches. ${ }^{5}$ Dietary management and weight loss are time-honored treatments, supported by several observational studies. ${ }^{6}$ Repeated LPs are sometimes used in patients with occasional symptom relapses, in pregnant women, or in the setting of rapidly declining vision to temporarily lower the CSF pressure while planning a more aggressive treatment. ${ }^{7}$

Acetazolamide is generally accepted as a first-line medication for lowering the intracranial hypertension in patients with IIH. Furosemide also has beneficial effects on CSF secretion and may be used. ${ }^{8}$ Other diuretics are used but no consistent therapeutic trend has been reported. Corticosteroids are not advocated for routine or longterm management of $\mathrm{IIH} .{ }^{9}$ The 
chronic headaches of IIH are best treated with conventional headache prophylaxis, although in some cases lowering the ICP with medical methods is effective. ${ }^{7}$ Because of the potential dangers, many authors do not advocate CSF shunting procedures for headache alone. At the same time, many of the agents used for headache prophylaxis in IIH (tricyclic antidepressants, selective serotonin reuptake inhibitors, sodium valproate, calcium channel blockers) may produce weight gain or edema that is undesirable in this population. ${ }^{10}$

Surgery is considered under the following circumstances: 1) progressive loss of vision despite maximal medical therapy; 2) severe or rapid visual loss at onset (malignant IIH), including the development of an afferent pupillary defect or signs of advancing optic nerve dysfunction; and 3) severe papilloedema causing macular edema or exudates. ${ }^{7}$ Surgical procedures used for the treatment of visual loss include optic nerve sheath decompression (ONSD) and CSF diversion procedures. Whether one procedure is superior to the other is controversial and the decision often depends on available resources and expertise. The success rate is comparable between ONSD and lumboperitoneal shunt. ${ }^{11} \mathrm{~A}$ CSF diversion procedure treats IIH by lowering ICP but requires insertion of a foreign body.

Lumboperitoneal shunting is more commonly performed than ventriculoperitoneal (VP) shunting because insertion and maintenance of patency may be more difficult in the latter procedure. However, VP and cisterna magna shunting may be successfully used. ${ }^{12}$

The complications of lumboperitoneal shunting are shunt valve or tubing obstruction, over-shunting (low pressure headache), catheter migration/abdominal pain, lumbar radiculopathy, infection, CSF leak, cerebellar tonsillar herniation (acquired Chiari malformation) and shunt dependency, but fortunately in our case, no complication was reported in one-year follow-up. ${ }^{13}$

\section{Conclusion:}

IIH is surprisingly a common disorder and often difficult to manage. Though lumboperitoneal shunt is commonly performed to manage this case, it was not done in our country. We have successfully managed this case and one-year follow-up revealed complete recovery. We have not faced any complication yet. So, it can be used further for IIH cases, which are difficult to manage.

\section{Conflict of Interest: None}

\section{References :}

1. Bandyopadhyay S. Pseudotumor cerebri. Arch Neurol 2001;58: 1699-701.

2. Durcan FJ, Corbett JJ, Wall M. The incidence of pseudotumor cerebri. Population studies in Iowa and Louisiana. Arch Neurol 1988; 45:757-7.

3. Susman JL. Benign intracranial hypertension. J Fam Pract 1990;30:290-2.

4. Friedman DI, Jacobson DM. Diagnostic criteria for idiopathic intracranial hypertension. Neurology 2002; 59:1492-5.

5. Frisén L. Swelling of the optic nerve head: a staging scheme. J Neurol Neurosurg Psychiatr 1982; 45:13-8.

6. Selhorst JB, Kulkantrakorn K, Corbett JJ, et al. Retinolbinding protein in idiopathic intracranial hypertension (IIH). J Neuroophthalmol 2000; 20(4):250-2.

7. Corbett JJ, Thompson HS. The rational management of idiopathic intracranial hypertension. Arch Neurol 1989; 46:1049-51.

8. Physicians' Desk Reference, 56th edition. Montvale, NJ: Medical Economics Company, Inc; 2002:2590-2595.

9. Liu GT, Kay MD, Bienfang DC, et al. Pseudotumor cerebri associated with corticosteroids withdrawal in inflammatory bowel disease. Am J Ophthalmol 1994; 117:352-7.

10. Friedman DI, Rausch EA. Headache diagnoses in patients with treated idiopathic intracranial hypertension. Neurology 2002; 58: 1551-3.

11. Rosenberg ML, Corbett JJ, Smith C, et al. Cerebrospinal fluid diversion procedures in pseudotumor cerebri. Neurology 1993; 43: 1071-2.

12. Howard J, Appen RE. Ventriculoperitoneal shunting for pseudotumor cerebri. Presented at the Association for Research in Vision and Ophthalmology, Fort Lauderdale, FL, 2002.

13. Deborah I. Friedman, MD and Daniel M. Jacobson, M. Idiopathic Intracranial Hypertension, J Neuro-Ophthalmol 2004;24: 138-145. 